\title{
Introduction
}

\section{Passing the Torch: An Introduction from New Editors-in-Chief Leisa Gibbons and Karen F. Gracy}

https://doi.org/10.1515/pdtc-2018-0008

\begin{abstract}
As the new editors of Preservation, Digital Technology and Culture, we welcome you to our first issue of 2018! We would like to thank our previous editor-in-chief Michèle Cloonan heartily for her contributions to the journal, and also give thanks for her mentorship during the editorial transition. In her final column as editor, Michèle wrote that she had witnessed much change in the preservation discourse over the previous five years. Discussion of technological and social issues across a range of topics and disciplines were given space in PDT\&C. The journal's coverage promoted a vision for a holistic and converged future of preservation that embodies multiple and varied ways of thinking and practice.
\end{abstract}

As editors-in-chief, we are continents apart; Karen resides in Ohio, USA, and Leisa lives in Western Australia. Yet, we feel that we are part of the change that Michèle has encouraged and documented and are inspired to build upon the foundations that she has laid. Earlier this year, we spent time identifying and consolidating our vision for the next 5 years. This work culminated in a call for papers, which lists emerging areas of interest for us, particularly in cultural heritage informatics, big data, indigenous knowledge, and personal archiving, as well as the varied uses and reuses of cultural heritage materials. Our goal is to showcase the multiple epistemologies of digital heritage and preservation, and how these can engage, support and ultimately enact social and cultural resilience and transformation.

We are pleased to present two interesting feature articles in this issue that embody these interests. Silvia Mazzetto's piece, "Heritage Restoration as a Tool to Promote Architectural Identity in the Gulf Regions," describes the challenges of constructing of a new architectural identity in Arab emerging cities such as Abu Dhabi in the United Arab Emirates, and Muscat and Salalah in
Oman, particularly the importance of protecting heritage sites of historical and cultural importance in the wake of the introduction of modern Western architectural styles. In Evanthia Samaras and Andrew Johnston's work, "Fleeting Film: Using Story to Seek Archival Permanence in the Transitory and Globalized Digital Visual Effects Industry," the authors consider how to apply archival principles to the preservation of multi-provenantial, multifaceted cinematic works that feature significant visual effects (VFX). The authors use storytelling as a method for documenting multinational VFX productions, describing the nature of the archival fonds, and guiding archival practice such as appraisal and selection. Both of these articles provide evidence of how social and technological changes are impacting traditional imperatives of archive and preservation work.

In addition to our featured research articles, we continue the journal's tradition of providing information about new ventures and events in the research and professional communities of cultural heritage via the Currents and Comments section. In this issue, we include a selection of news items from around the world, which report on projects and activities that expose hidden and silenced information, celebrate shared heritage, deliver innovative visions, and challenge how we document cultural and social change. We welcome submissions for inclusion to the Currents and Comments section from our readers; please send your news to us at eics.pdtc@gmail.com for inclusion in future issues.

We also are pleased to introduce a new occasional feature for this issue, called Beyond Findings: Conversations with Experts. Beyond Findings is a place to learn something different about cutting-edge research and projects beyond that of a traditional research report or journal article. Delivered in a short interview style, Beyond Findings provides an opportunity to ask questions about what projects may mean to our audience. In this issue, we talk to Dr. Pauline Joseph from Curtin University in Australia, who works with digital visualisation of data and cultural heritage. The interview explores the nature 
of 3D immersive technologies and how they can be used to interrogate massive, complex datasets documenting the information seeking behavior of university students. We welcome requests for interviews and submissions of interviews for this new section as well.

We encourage our readership to continue this journal with us to explore new frontiers in preservation and heritage work, and look forward to hearing from you as readers and contributors.

Sincerely,

Leisa Gibbons and Karen F. Gracy

Editors-in-Chief

Preservation, Digital Technology \& Culture 